\title{
Clima de convivencia escolar en Chile: un análisis desde el nuevo marco de medición de calidad educativa
}

Padilla Fuentes, Geraldo; Rodríguez Garcés, Carlos

Clima de convivencia escolar en Chile: un análisis desde el nuevo marco de medición de calidad educativa

Revista Educación, vol. 43, núm. 2, 2019

Universidad de Costa Rica, Costa Rica

Disponible en: http://www.redalyc.org/articulo.oa?id=44058158045

DOI: https://doi.org/10.15517/revedu.v43i2.34117

Esta obra está bajo una Licencia Creative Commons Atribución-NoComercial-SinDerivar 3.0 Internacional. 


\section{Clima de convivencia escolar en Chile: un análisis desde el nuevo marco de medición de calidad educativa}

\section{School Coexistence in Chile: An Analysis Based on the New Educational Quality Measurement Framework}

Geraldo Padilla Fuentes

Universidad del Bio-Bio, Chile

gpadilla@ubiobio.cl

iD http://orcid.org/0000-0003-0882-1818

Carlos Rodríguez Garcés

Universidad del Bio-Bio, Chile

carlosro@ubiobio.cl

iD http://orcid.org/0000-0002-9346-0780
DOI: https://doi.org/10.15517/revedu.v43i2.34117

Redalyc: http://www.redalyc.org/articulo.oa?id=44058158045

Recepción: 21 Enero 2019

Aprobación: 27 Junio 2019

\section{Resumen:}

Los puntajes en pruebas estandarizadas y el rendimiento escolar se han tornado indicadores insuficientes para medir y evaluar calidad en procesos de enseñanza-aprendizaje. En este contexto, los Otros Indicadores de Calidad (OIC) definidos por el Ministerio de Educación en Chile buscan subsanar esta debilidad complementando la métrica tradicional con un conjunto de índices asociados al desarrollo personal y social del estudiantado. Objetivo: perfilar el comportamiento del Clima de convivencia escolar y sus dimensiones, constituido en uno de los OIC trascendentales para dar cuenta de las condiciones en que se suscita el aprendizaje. Método: utilizando cuestionarios de contexto aplicados a estudiantes, profesores/as, padres y madres se analizan percepciones sobre organización, seguridad y respeto del espacio educativo con modelamiento estadístico inferencial. Resultados: la percepción y evaluación que se hace de la situación de convivencia tanto escolar como áulica está determinada por el rol, posición y responsabilidad del agente evaluador, ello explicaría la perspectiva crítica del estudiantado, la neutralidad docente y la desvinculación parental con el clima relacional del acontecer educativo. En razón de su intrínseca naturaleza de cotidianidad y compartir forzado en un espacio físico reducido y de proximidad, el clima áulico es particularmente problemático. Conclusiones: la problematización de este OIC realza la necesidad de fortalecer el educar para vivir en comunidad, particularmente cuando las percepciones de los actores están fuertemente colonizadas por la deseabilidad social y naturalizadas por la cotidianidad de climas de convivencia deteriorada.

Palabras Clave: Calidad de la educación, Clima de convivencia escolar, Clima áulico, Otros Indicadores de Calidad, Comunidad escolar.

\section{Abstract:}

Scores on standardized tests and school performance are inadequate indicators that have been used to measure and assess quality for teaching-learning processes. Given this context, Other Quality Indicators (OQI) defined by Chile's Ministry of Education seek to remedy this weakness by supplementing traditional metrics with a group of indexes associated with personal and social development of the students. Objective: Draw a profile of the trend for school coexistence climate and its dimensions comprised by a cross-cutting OQI to report on conditions that foster learning. Method: Student, Teacher and Parent questionnaires addressing school context analyzing perceptions about organization, safety of contextual questionnaires and respect of the educational space with inferential statistical modelling. Results: Perception and assessment about the state of coexistence in the school and classroom is determined by the role, position and responsibility of the evaluator. This explains the critical perspective of the students, the impartiality of the teachers and disassociation of the parents with the status of contexts of relationships at school. Given the intrinsic nature of everyday life and forced sharing of reduced physical spaces, classroom relationships are particularly problematic. KEYworDs: Quality of Education, School Coexistence Climate, Classroom Climate, Other Quality Indicators, School Community. 


\section{INTRODUCCIÓN}

Como institución, el quehacer educativo persigue sustancialmente dos objetivos: educar y socializar; ambos en pos de asegurar la inserción y adaptación de los ciudadanos al medio social que los contiene (Gilbert, 2012). La responsabilidad por asegurar acceso al servicio y Derecho educativo recae sobre el Estado y su compromiso con el bienestar material y subjetivo de las personas, debiendo velar por el constante crecimiento de los niveles educativos como vía para demostrar progreso y desarrollo (ONU, 2010). Los sistemas educativos contemporáneos son fruto de sucesivas transformaciones, ajustes y mejoras a los principios que rigen la pedagogía y definición de lo necesario para aprender a desenvolverse y vivir en comunidad, lo que se traduce en criterios de cobertura, equidad, calidad, profundidad y gestión.

En este sentido, el promedio de notas y los resultados en pruebas estandarizadas se posicionaron tempranamente como indicadores de calidad de la educación entregada. A través del análisis de puntajes y notas se establecían los niveles de eficiencia y eficacia del sistema teniendo a la base un programa general o currículum que definía el transcurrir de los ciclos educativos (García y Pérez, 2008). Este enfoque, desarrollado en la década de los 60 y 70 principalmente en Estados Unidos y Europa, centra su preocupación en los resultados de pruebas estandarizadas y las debilidades que evidencia el sistema en el logro de la promoción de aprendizajes para una población escolar supuestamente homogénea. Un hito en este campo fue el conocido Informe Coleman (1968), que develó la importancia que características de origen social y familiar tienen por sobre las de un centro educativo en la explicación del rendimiento escolar (Grade Point Average); conclusiones que pese a su relevancia han sido marginalmente asimiladas a la política educativa (Carabaña, 2016).

Bajo este paradigma, en 1988 Chile crea el Sistema de Medición de Calidad de la Educación (en adelante SIMCE), y junto con ello instala un mecanismo de pruebas estandarizadas el cual pretendía recoger mediante un único indicador la calidad del aprendizaje alcanzado por el estudiantado chileno. Comprensión de calidad confinada a una perspectiva de productos que jerarquizaba unidades educativas con base en el puntaje exhibido e informaba al público cuál era la mejor opción escolar entre las disponibles. Esta imagen simplificada y reduccionista de la calidad educativa, hegemónica por más de 20 años, comienza a ser profundamente cuestionada en su capacidad para develar la real situación de los colegios cuando la perspectiva compleja empieza a ser parte de la política educativa.

\section{El porqué de los Otros Indicadores de Calidad}

A poco tiempo de ponerse en marcha, SIMCE enfrentó varias críticas por parte de la comunidad escolar y académica, quienes acusaban que medir calidad no podía consistir en comparar resultados que de partida eran imposibles de alcanzar con igualdad entre las instituciones escolares, pues tanto los recursos materiales como pedagógicos y administrativos no se encontraban repartidos con equidad (Martinic, 2002). También se denunció reiteradamente una alta correlación entre capital educativo y rendimiento alcanzado, donde más que Derecho, la calidad medida parecía un privilegio de algunos establecimientos escolares asociado a clases privilegiadas (Cornejo y Redondo, 2007); así mismo, reducir la estimación de calidad al producto de un test ocultaba la importancia que tienen los valores e ideas propugnados por la escuela para el futuro del alumnado (Serramona, 2004). Por último, hubo quienes revisando reportes SIMCE descubrieron numerosas imprecisiones técnicas en elaboración de las pruebas, la consistencia de las preguntas y decisiones metodológicas, todo lo cual hacía parecer al proceso una caja negra abierta a múltiples interpretaciones (Flórez, 2013). En síntesis, la perspectiva de SIMCE fortalecía un concepto de calidad el cual no ponía en duda la segmentación presente en el sistema educativo, además de no considerar que la escuela también es responsable por instalar competencias que tributen al desarrollo personal y social en el alumnado, como 
son participación y formación ciudadana, equidad de género y/o capacidad para contribuir a un clima de convivencia seguro, organizado y respetuoso.

En el año 2009, y bajo lo que se puede considerar una primera aproximación al paradigma de pensamiento complejo y su preocupación por el fenómeno educativo desde la integralidad (Pereira, 2010), se promulgó en Chile la Ley General de Educación que estableció una serie de reformas al funcionamiento del sistema educativo en lo tocante a requisitos mínimos a exigir por nivel educativo, las normas que permitirían al Estado velar que se cumplan tales objetivos y los requisitos que definirían el reconocimiento oficial de los establecimientos en todo el sistema. En cuanto a calidad, se reconocía el rol del Estado en la provisión y aseguramiento de esta en el sector educativo, con tres medidas clave: establecer Estándares de Aprendizaje por nivel educativo que expresan aprovechamiento de currículum; rediseño del Currículum Escolar según Bases Curriculares específicas por ciclo (parvulario, básico y secundario); y modificaciones al modo de operar que tenía SIMCE, concretamente su asignación como herramienta a cargo de la Agencia de Calidad de la Educación, institución creada en el año 2011, la cual forma parte del Sistema Nacional de Aseguramiento de la Calidad. Esta agencia también debe evaluar los indicadores de desarrollo personal y social del estudiantado (IDPS, también llamados Otros Indicadores de Calidad) creados para complementar la medición académica con un conjunto de competencias que la escuela debería promover en estudiantes para dotar de calidad su quehacer (Ministerio de Educación de Chile, 2009). Los OIC o IDPS son Autoestima académica y motivación escolar; Participación y formación ciudadana, Hábitos de vida saludable, Asistencia escolar, Retención escolar, Equidad de género, Titulación técnico-profesional y el que resulta de interés para esta investigación: Clima de convivencia escolar.

La nueva escuela aparece ahora como un espacio multidimensional saturado de fenómenos a considerar y valorar para definir el grado de calidad atribuible a los procesos educativos en que participa el alumnado. En la mejora de su funcionamiento, el quehacer educativo requiere de la participación de administradores, profesionales y asistentes de la educación (docentes, auxiliares, psicólogos, entre otros), alumnos/as, padres y madres, tanto en las aulas como los espacios compartidos de convivencia en el colegio (Ministerio de Educación de Chile, 2014).

\section{El Clima de convivencia escolar como objeto de intervención}

Con un set de nuevas reglas y la presión por ampliar el modo de estimación de calidad, las intervenciones de la política educativa, administraciones públicas y profesionales de la educación ampliaron el foco, número y profundidad de sus estimadores. Si previo a la formalización de los OIC la preocupación era el déficit de cobertura curricular, ahora se planteaba un panorama complejo donde importa el hecho integral que se desarrolla en las aulas, es decir, el apoyo a procesos democráticos, el estímulo a la motivación escolar, la reafirmación pedagógica de la autoestima académica y cuáles son las condiciones en que se urden las relaciones interpersonales entre los actores de la comunidad escolar (Ministerio de Educación de Chile, 2013). En términos de clima de convivencia, lo que importa para este OIC es configurar la calidad del escenario donde se aprende a conocer, a ser y vivir juntos según la perspectiva de estudiantes, profesores/as, padres y madres, recogiendo sus valoraciones en cuestionarios de contexto que acompañan las aplicaciones de las pruebas SIMCE. El clima aparece como un campo de estudio e intervención del modo y lugar en que convive y aprende el alumnado, resultado de las interacciones entre los agentes de la comunidad educativa y su organización, respeto y seguridad.

La inquietud por incluir este OIC como criterio de calidad reside en un problema persistente para el sistema educativo nacional y es que, según diversos resultados de investigación, las manifestaciones de violencia están correlacionadas con la condición de vulnerabilidad económica y educativa del alumnado (Leyton, Vásquez y Fuenzalida, 2012; Guerra et ál. 2011). Esto menoscaba la efectividad y profundidad de las intervenciones pedagógicas, pues presiona por el desvío de los esfuerzos en el aula desde la puesta y diálogo 
de contenidos hacia el reforzamiento de medidas de control, especialmente a cargo del y la docente. Por lo demás, los episodios de violencia mitigan la seguridad entre estudiantes y suelen afectar el rendimiento y niveles de logro, ocasionan reactividad a los estímulos y exacerban los sentimientos de frustración (Carrasco y Trianes, 2010; Conejeros, Rojas y Segure, 2010; Barca, Almeida, Porto, Peralbo y Brenlla, 2012). En nuestro país, resultados como estos han sido pesquisados por instituciones como el Ministerio del Interior (2006) e investigadores como Trautmann (2008) y Tijmes (2012), mientras que a nivel latinoamericano estudios de UNESCO y CEPAL han reportado que violencia, agresiones verbales y reprochamiento cruzado entre profesores/as y estudiantes son hechos transversales e indeseables en los sistemas educativos (Cervini, Dari y Quiroz, 2015; Román y Murillo, 2011; López et ál. 2011).

Por esto, en un contexto de reformas y expansión a la composición, operacionalización y evaluación de la calidad en educación, el Clima de convivencia escolar destaca con legitimidad, por cuanto es donde se desarrollan los procesos educativos y que debe operar con independencia de la posible segmentación socioeducativa. En sintonía, el objetivo de este trabajo es perfilar el comportamiento que tiene el OIC Clima de convivencia escolar (CCE) según los alcances que permite el SIMCE desde una perspectiva compleja. Para ello se hace uso de los cuestionarios de contexto que acompañan las pruebas SIMCE y son expresión de la valoración que profesores/as, estudiantes y apoderados/as hacen de los espacios áulicos y escolares que comparten. Así mismo, este análisis se sitúa entre las primeras mediciones del OIC, teniendo como referencia el segundo año de medición del estado del clima de convivencia (Ministerio de Educación de Chile, 2014), por lo que los aportes efectuados pueden considerarse 'preliminares' del nuevo marco de calidad educativa en Chile, relevantes para la toma de futuras decisiones como para la discusión general que concita la mejora del vivir común entre académicos, profesionales de la educación u otros interesados. Los matices en la presentación de resultados están marcados por la comparación de valoraciones por tipo de actor, niveles educativos incluidos, espacios de convivencia y dimensiones que la componen: organización, seguridad y respeto.

\section{Metodología}

\section{Diseño}

Este artículo se posiciona desde una perspectiva cuantitativa tanto en lo que refiere a los procedimientos de muestreo representativos, levantamiento de información con protocolos estandarizados y técnicas estructuradas como son los cuestionarios de contexto aplicados a estudiantes, profesorado, padres y madres; datos que son procesados y representados a través de índices y tablas resumen, con ocasión de los análisis estadísticos descriptivos e inferenciales desarrollados para estimar las valoraciones que estos agentes de la comunidad educativa tienen y hacen de la convivencia escolar.

\section{Muestra}

Al ser un proceso censal, el Sistema de Medición de la Calidad de la Educación (SIMCE) recoge información de la totalidad de los establecimientos del país y gran parte de los miembros de la comunidad escolar. Los actores considerados en este análisis son los y las estudiantes, padres, madres y profesores/as de un subsector específico del currículum (Matemáticas) pertenecientes a los niveles educativos de $8^{\circ}$ básico (Primaria) y $2^{\circ}$ Medio (Secundaria), correspondientes a los procesos 2014. Dados los requerimientos métricos de equivalencia a nivel de ítems y escala, estos grados educativos dan mayores garantías en razón de la maduración lingüística del estudiante y comprensión verbal del sentido de los ítems. Además, al pertenecer a niveles 
escolares distintos se hace posible analizar comparativamente diferencias y similitudes en las percepciones que tienen del clima en que conviven.

En el año 2014, y con independencia del nivel educativo en que se encuentren, son alrededor de cuatrocientos mil (401.469) estudiantes que rinden SIMCE. Dada la conformación del sistema escolar chileno, que presenta una mayor matrícula en el nivel de educación básica, se registra una natural menor presencia de estudiantes de enseñanza media.

Con independencia relativa del nivel educativo analizado, se observa una gran proporción de alumnos/ as con bajo desempeño SIMCE en el área de matemáticas, con un 38,3\% en Octavo Básico (Pje <297) y un $45,4 \%$ en Segundo Medio (Pje <294) no obtienen un nivel de logro suficiente para el nivel en que se encuentran. La baja calidad del sistema educativo, sus segmentados niveles de logro y la incapacidad que tiene la escuela de revertir las desigualdades sociales adscritas, se constituyen en características distintivas del sistema escolar chileno (Tabla 1).

TABLA 1

Caracterización de estudiantes que rinden SIMCE 2014 (\%)

\begin{tabular}{|c|c|c|c|c|c|}
\hline & $8^{\circ} \mathrm{B}$ & $2^{\circ} \mathrm{M}$ & & $8^{\circ} \mathrm{B}$ & $2^{\circ} \mathrm{M}$ \\
\hline Sexo Hombre Mujer & $49,849,9$ & $50,549,5$ & Sexo profesor Hombre Mujer & $48,751,3$ & $42,357,7$ \\
\hline Dep. Admin. Municipal Part. Subv Part. Pagado & $28,762,48,9$ & $41,350,97,8$ & Educación apoderados /as Hasta básica Hasta media Superior C/Inc & $13,849,236,9$ & $15,149,435,4$ \\
\hline Nivel de logro Insuficiente Elemental Adecuado & $38,347,014,7$ & $45,438,516,1$ & GSE Bajo-Medio Bajo Medio Medio Alto-Alto & $52,525,022,5$ & $46,431,324,3$ \\
\hline
\end{tabular}

\begin{abstract}
Elaboración propia basada en datos aportados por Agencia Calidad de la Educación (2014). $8^{\circ} \mathrm{b}=$ Octavo Básico; $2^{\circ} \mathrm{m}=$ Segundo Medio; Dep= Dependencia; Admin= Administrativa; Part = Particular; Subv= Subvencionado; GSE= Grupo Socioeconómico del establecimiento; $\mathrm{C} /$ Inc= completa/incompleta.
\end{abstract}

En cuanto a las características de padres y madres, alrededor del 40\% reporta más de 12 años de escolaridad para ambos niveles educativos. Respecto del profesorado, se registra una presencia levemente mayor de mujeres, que se acentúa en segundo medio (57,7\%).

La unidad de análisis exhibe una predominancia de estudiantes de niveles socioeconómicos bajo y mediobajos ( $52,5 \%$ en $8^{\circ}$ básico y $46,4 \%$ en $2^{\circ}$ medio) y de establecimientos particular subvencionados $(62,4 \%$ en $8^{\circ}$ básico y $50,9 \%$ en $2^{\circ}$ medio). Tiende a ser una constante histórica la pérdida progresiva de matrícula del sector público como resultado de la profunda segmentación socioeducativa, por lo cual las escuelas son espacios donde la heterogeneidad económica y social es escasa. Importantes contingentes en situación de vulnerabilidad conviven con pares en igual condición y similares formas de pensar, sentir y actuar.

\title{
Instrumento
}

La investigación hace uso de las encuestas de contexto que acompañan las pruebas SIMCE, las cuales son cumplimentadas por estudiantes, profesores y apoderados/as. Estos son cuestionarios de información autorreportada que recogen la percepción que tienen los distintos actores de la escena educativa. En lo específico, se abordan los ítems vinculados al clima de aula y del establecimiento.

El proceso 2014 sirve de referencia para el análisis de los cambios que la nueva medición de la calidad educativa introdujo en el abordaje del clima de convivencia. En este proceso algunos ítems de los cuestionarios de contexto fueron adecuados, se mejoró su redacción y profundizó en otras dimensiones. A modo de ejemplo, en el 2014 más allá de la sencilla consulta respecto de la existencia en el establecimiento de un plan de convivencia, tal como se hacía en 2010, la indagatoria ausculta si estas reglas son compartidas, conocidas y respetadas por el profesorado y el alumnado. La validez de estos instrumentos está sustentada en su carácter oficial y personal, por lo que sería reflejo de lo que cada actor piensa en forma sincera y situada. 
La medición de la seguridad en estudiantes se ha vuelto hacia lo percibido más que lo presenciado, por lo que pregunta sobre cómo o cuán seguro se siente en el patio de la escuela, los baños, el comedor, y si existen prácticas de riesgo como consumo de alcohol y/o drogas en el establecimiento. En el caso del profesorado se les pregunta sobre la relación con otros colegas, resolución de conflictos y redes de apoyo en caso de algún problema.

El asunto de padres y madres es especial, puesto que aun cuando la vida escolar les hace participar con determinada frecuencia y compartir con otros/as apoderados/as, resulta no del todo satisfactorio el enfoque tan pragmático que tiene su cuestionario. La evaluación del ambiente seguro que se les permite está referida a criterios de si es buen lugar para el/la estudiante, si le gusta asistir, si lo hace tranquilo/a y se siente protegido/ a junto a sus compañeros, no acerca de si en el aula su hijo/a dice tener problemas, sentirse intimidado/a o no puede resolver constructivamente conflictos con sus compañeros.

\section{Procedimiento}

Procurando subsanar las limitaciones que dificultan el establecimiento de una métrica de funcionalidad equivalente para fines comparativos, se construyeron diversos índices que serían expresión de la percepción y actitud que estudiantes, profesores/as y apoderados/as tienen respecto a la Seguridad, Organización y Respeto del clima en que conviven, distinguiendo según pertenezcan a Octavo Básico o Segundo Medio.

Originalmente, la Agencia de Calidad no distingue entre aula o establecimiento, sino entre grados y ciclos educativos. Este trabajo lo hace, por cuanto la opinión respecto a situaciones de inseguridad física o emocional, falta de normas o procedimientos que faciliten la vida en común y actos que fomenten la discriminación, varían según la proximidad que tengan los eventos al agente evaluador. Además, la estimación del control que se tiene sobre cada espacio atenúa o potencia la sensación de riesgo, y las relaciones que forjamos con quienes compartimos constantemente es mucho más fuerte en el aula.

Los ítems de cada cuestionario fueron agrupados según espacio (aula-establecimiento) y ambiente (seguridad-organización-respeto) al que estén circunscritos, siguiendo desde un principio los criterios y definiciones que entrega la Agencia de Calidad en sus informes:

- Clima de convivencia: percepción que estudiantes, profesores/es y apoderados/as tienen respecto a la existencia de un ambiente de respeto, seguro y organizado en el establecimiento.

- Ambiente Organizado: existencia de normas de convivencia claras, conocidas, exigidas y respetadas por todos los miembros de la comunidad escolar, junto al predominio de mecanismos constructivos de resolución de conflictos.

- Ambiente de Respeto: Trato respetuoso entre miembros de la comunidad, la valoración de la diversidad y la ausencia de discriminación. Además, considera las percepciones de cuidado del establecimiento y su entorno.

- Ambiente Seguro: Grado de seguridad y de violencia física y/o psicológica en el establecimiento, además de la existencia de mecanismos para prevenir y actuar frente a la violencia escolar.

Así, lo que se obtiene al final son cuatro indicadores: Clima de convivencia, Ambiente de respeto, Ambiente seguro y Ambiente organizado, los cuales pueden desagregarse por nivel (Octavo Básico y Segundo Medio).

A fin de permitir la comparación intra e inter conglomerados, y resguardando la orientación de los ítems, se ejecutó un proceso de estandarización de las puntuaciones obtenidas en las dimensiones identificadas, lo que deriva en un continuo que transita de 0 a 1 , donde más cercano al entero significa mejor percepción y actitudes favorables hacia el clima de convivencia y dimensiones que la integran. 


\section{Resultados}

La calidad educativa como constructo teórico práctico de carácter complejo encuentra en el clima de convivencia su referente más significativo. La escuela, en tanto institución educativa que busca responder a los requerimientos que la modernidad le impone, debe procurar la instalación de competencias procedimentales y actitudinales que permitan vivir en sociedad. En consecuencia, transversalmente a la generación de habilidades cognitivas el ideal pedagógico demanda centralizar la preocupación por el desarrollo del sentido de pertenencia, participación ciudadana, autoestima académica, tolerancia y respeto a otros.

La convivencia escolar es un fenómeno heterogéneo en razón de la composición, subsistemas y estructuras que la delimitan y caracterizan. Es así que, aun compartiendo una misma unidad educativa, estudiantes, profesores/as y apoderados/as pueden diferir en su evaluación con base en sus experiencias particulares, significados que le otorgan y rol que juegan en cada subsistema.

Como informa la Tabla 2, con independencia de la nomenclatura utilizada en las ponderaciones para la construcción del índice de convivencia (global original/global modificado), se reporta un clima escolar más deteriorado en Segundo Medio. Analizado desagregadamente según el rol del agente de la comunidad educativa, el estudiantado aparece como el actor más crítico en la evaluación del clima en que conviven [F $(1)=348,355 \mathrm{p}<, 01]$, percepción más deteriorada entre quienes cursan segundo año de enseñanza media $\left[t_{r e l}\right.$ $(2164)=5,903, \mathrm{p}<, 01]$, aun cuando sea pequeño el tamaño del $[\mathrm{d}=, 122]$. En comparación al ciclo básico, en el estudiantado secundario el abanico de interacciones se amplía, añaden nuevos intereses a sus relaciones y se comportan más orientados por el pensamiento emocional (Fueyo, Martín y Dapelo, 2010), haciendo más complejas las dinámicas de convivencia y resolución de conflictos, particularmente en espacios educativos vulnerables. No obstante, esta evaluación puede transitar más allá de la simple constatación objetiva de un hecho para constituirse en la expresión perceptiva de un agente que, dada la nueva etapa del desarrollo personal y social en que se encuentra, tiene mayor sensibilidad y capacidad para advertir situaciones violentas, desorganización o discriminación, antes relativizadas.

TABLA 2

Percepción general del clima de convivencia de los actores según nivel educativo

\begin{tabular}{l|l|l|l|l|l|}
\multicolumn{2}{c}{ Profesorado } & Estudiantes & Apoderados & $\begin{array}{l}\text { Global } \\
\text { original }\end{array}$ & $\begin{array}{l}\text { Global } \\
\text { modificado** }\end{array}$ \\
\hline $8^{\circ}$ Básico &, 735 &, 710 &, 726 &, 727 &, 730 \\
\hline $2^{\circ}$ Medio &, 709 &, 683 &, 730 &, 709 &, 705 \\
Global actor &, 732 &, 718 &, 729 & & \\
\hline
\end{tabular}

\footnotetext{
Elaboración propia basada en datos aportados por Agencia Calidad de la Educación (2014).

* Índice calculado según ponderaciones de la Agencia de Calidad de

la Educación (estudiantes*,50; profesores/as*,10; apoderado*,40)

** Índice calculado según ponderaciones ajustadas (estudiantes*,50; profesores/as*,40; apoderado*,10)
}

En el cálculo del OIC-Clima de Convivencia, la Agencia de Calidad prioriza como agente informante a padres y madres, con una ponderación muy por sobre la asignada al profesorado ( $40 \%$ vs. 10\%) y solo levemente inferior a la relevancia estadística otorgada al contingente de estudiantes (50\%). Ponderación diferenciada en razón a la importancia que tendría la implicación parental en el diseño y ejecución de estrategias para optimar la convivencia. Analizados los datos, estos reportan que son precisamente los padres y las madres quienes tienden a exhibir una menor concordancia con el alumnado al evaluar climas de convivencia, siendo significativamente más favorables en su percepción. Con independencia del rol que juega la implicación parental educativa en los procesos de aprendizaje, en lo que a convivencia respecta ellos parecieran ser los agentes menos informados. No solo es recurrente la queja entre el profesorado 
por su falta de involucramiento y acompañamiento pedagógico al estudiante en el hogar (Gázquez, Pérez, Acién y Fernández, 2009; Del Rey, Ortega y Feria, 2009; Cornejo y Redondo, 2007), sino que además su preocupación por los ambientes de interacción se activa esporádicamente y por contingencias que escalaron en su gravedad.

Al desglosar el clima de convivencia en ambientes (Seguridad, Respeto, Organización) se acentúa la menor evaluación que hace el estudiantado de Segundo Medio respecto de quienes asisten a Octavo Básico (Tabla 3). Para quienes ya están en secundaria, el Ambiente Organizado está deteriorado, carente de reglas para la vida común que sean conocidas y respetadas por los miembros, o bien escaso de mecanismos constructivos para resolver conflictos. La percepción que proyectan sobre inseguridad física y/o emocional permite inferir que enfrentan un déficit para mantener un clima de convivencia estable y duradero donde predominen las herramientas dialógicas para la resolución de conflictos.

El estudiantado de Segundo Medio parece convivir con varias dificultades, sin reglas o marcos claros que exijan a todos resolver situaciones de conflicto en forma dialógica o consensuada. De allí la vulnerabilidad percibida al compartir espacios crimípetos que en su morfología pueden volverse inseguros (San Juan y Vozmedia, 2008), y las dudas respecto a qué hacer para prevenir o enfrentar la violencia, particularmente en lugares alejados de la vigilancia de profesores/as o inspectores/as. Este diagnóstico secular parece estar delimitado por las disposiciones institucionales que deberían velar por la vida común y su regulación. Al ser consultados por las normas de convivencia en el establecimiento, el alumnado expresa un significativo desacuerdo respecto a que estas sean conocidas por todos sus compañeros (47,8\%), se apliquen de forma justa $(39 \%)$ y que se respeten $(31,9 \%)^{[1]}$. Complementario, reportan que en sus establecimientos ocurren al menos una vez por mes agresiones verbales $(60,3 \%)$, peleas (32,5\%), prácticas de aislamiento social (31\%), robos $(25,9 \%)$ y daños al mobiliario $(25,2 \%)$.

Por su parte, y en sintonía con lo planteado por estudiantes, padres y madres concuerdan en que los ambientes escolares de enseñanza media deben lidiar con violencia a menudo, o que cuando la advierten no cuentan con los procedimientos oportunos para prevenirla y enfrentarla. En efecto, un $40,1 \%$ informa la manifestación de insultos, garabatos y descalificaciones con habitualidad al interior del establecimiento, así como la presencia de bullying (47,1\%). Conflictos recurrentes que alteran las condiciones de seguridad física y emocional del espacio de convivencia a pesar de que los mismos apoderados reportan que se han difundido normas para prevenirlo $(81,3 \%)$, tomado medidas frente a las denuncias efectuadas $(82,1 \%)$ y se les ha involucrado en la prevención y reacción $(78,9 \%)$. Además, mayoritariamente dan cuenta de que el trato hacia estudiantes es respetuoso (91,7\%), de igual modo que el de estos hacia el personal docente (80\%) y entre ellos mismos (76,2\%). Positiva evaluación del ambiente de Respeto en clara contradicción con lo informado sobre Seguridad, por cuanto se esperaría una fuerte sintonía en dimensiones asociadas al mismo constructo clima de convivencia; disonancia explicada por el reducido grado de participación que tienen en la cotidianidad escolar en comparación con docentes y estudiantes, con una implicación que se releva frente a situaciones de mayor connotación.

Debido al funcionamiento escolar tradicional, la participación de padres o madres está ajustada por labores de control y verificación de sus hijos/as, debiendo encargarse de asistir a reuniones mensuales de curso y aparecer en caso de ausencia a clases o mal rendimiento. Con esto, los escenarios que observa son artificialmente ordenados o tranquilos; salas donde solo ve a otros padres, inspectorías donde cada quien ocupa su puesto y cumple una tarea, oficinas de atención escolar donde cada cosa tiene su sitio. Los padres no ven el espacio escolar de la misma forma que sus hijos e hijas, no se alojan en la cotidianidad del recreo o los pasillos llenos, el comedor bullicioso, escenarios donde efectivamente ocurren los incidentes. Por su parte, las extensas jornadas de trabajo, la masificación de herramientas individuales para el uso de la tecnología, la irrupción del ocio fuera de casa como parte del habitus (Bourdieu, 2016) y las características propias de la etapa adolescente dificultan la comunicación entre padres e hijos/as, limitando las transferencias de información a breves inputs y outputs por la mañana y noche. Todos estos factores, usualmente escindidos de 
lo que consideramos como parte de la dinámica educativa (Cornejo y Redondo, 2007), afectan la vida común que entablan los y las estudiantes en los niveles secundarios, alejados además de la óptica que sus padres tienen al respecto.

TABLA 3

Percepción de ambientes que componen clima de convivencia según nivel educativo

\begin{tabular}{|c|c|c|c|c|c|c|}
\hline & \multirow{2}{*}{\multicolumn{3}{|c|}{$2^{\circ}$ Medio }} & \multirow{2}{*}{\multicolumn{3}{|c|}{$8^{\circ}$ Básico }} \\
\hline & & & & & & \\
\hline & Estudiante & Profesor & Apoderado & Estudiante & Profesor & Apoderado \\
\hline Organizado Respeto Seguro & $, 626,695,666$ & $, 645,788,702$ & $, 774,850,608$ & $, 708,721,697$ & $, 679,796,721$ & $, 659,840,754$ \\
\hline
\end{tabular}

Elaboración propia basada en datos aportados por Agencia Calidad de la Educación (2014).

Analizada la percepción del profesorado según el Tabla 3, se reporta el Ambiente Organizado como dimensión crítica, particularmente en el nivel educativo de Segundo Medio, aun cuando son ellos quienes velan por el cumplimiento de normas y reglas, cuya aplicación contribuye in situ a la resolución de conflictos. El Ambiente Organizado es la dimensión del Clima de Convivencia más sujeta a control por parte del profesor, resultando sorpresiva su deficiente evaluación. Particularidades del rol pedagógico que los define como el único actor de la comunidad educativa que transita cotidianamente entre los distintos espacios de interacción, por lo que su percepción puede variar considerablemente respecto del aula, sitio donde es responsable directo, y el establecimiento, lugar de responsabilidad más diluida.

Sobre la situación organizacional de los colegios, gran parte del profesorado de segundo medio declara que las normas de convivencia y disciplina son conocidas por toda la comunidad escolar $(87,1 \%)$, el proyecto educativo está ampliamente difundido (80\%), los objetivos de la planificación para el aprendizaje se cumplen $(74,7 \%)$ y las normas de convivencia se aplican siempre con el mismo criterio (68,9\%). Diagnóstico que pone por descarte la atención en la sala de clases como sitio caótico o de comportamiento laissez-faire. Aquí, una de las mayores quejas pasa por el retraso de estudiantes en el reingreso al aula luego del recreo o cambio de hora (45,8\% dice que sucede siempre o muchas veces), lo que interrumpe el trabajo de los primeros minutos. Captar la atención del grupo es sustancial para vincular los contenidos a revisar con lo hecho la clase anterior, entregar instrucciones que faciliten las actividades propuestas y generar un espacio óptimo donde nadie se retrase; tarea que resulta complicada para 1 de cada 4 profesores/as, ya sea porque los y las estudiantes se distraen o molestan a otros compañeros $(26,9 \%)$, o porque deben interrumpir la clase para hacer callar o reprenderlos verbalmente (29,3\%). Con esto, el profesorado da cuenta de una percepción crítica del clima áulico, casi tan aguda como la de sus estudiantes, aunque atribuible a razones distintas. Aun cuando tienen trayectorias escolares extensas que posibilita la interiorización de reglas y sanciones de convivencia, cerca de la mitad de los y las estudiantes acusa desconocerlas (47,8\%), mientras que un 32,1\% de sus docentes percibe que estas no son respetadas.

En las otras dos dimensiones del clima de convivencia, Respeto y Seguridad, las evaluaciones mejoran, a pesar de que su comportamiento difiera según actor y nivel educativo. En el caso de Segundo Medio, al ser consultados sobre el trato respetuoso entre miembros de la comunidad, solo un $66,1 \%$ menciona estar satisfecho con las relaciones interpersonales que mantiene en la escuela, mientras que la gran mayoría de sus profesores/as (97,4\%) asevera que entre grupos predomina la comunión. Débil sintonía entre las opiniones que entregan los dos tipos de actor que mayor tiempo comparten la rutina escolar, más sugerente si se compara con la de padres, según quienes el respeto sería conditio sine qua non del regular funcionamiento escolar. Así, una proporción voluminosa de estos últimos considera que el profesorado vela por el buen trato, tanto hacia ellos en calidad de apoderados/as (96,8\%) como entre los y las estudiantes que atienden (91,7\%); además, manifiestan que como padres promueven los vínculos respetuosos (95,3\%). Sin embargo, esta percepción disminuye al evaluar el comportamiento del alumnado hacia sus docentes ( $80 \%$ ) y entre compañeros de curso 
(76,2\%), lo cual sería señal de una actitud parental condescendiente con quien percibirían como autoridad en el espacio escolar, y austera con quienes parecen no tomar la responsabilidad que les corresponde en razón de la edad en que se encuentran.

En cuanto a la dimensión de Seguridad, 2 de cada 5 estudiantes de Segundo Medio (40,2\%) manifiesta que sufre u observa violencia verbal cada semana o cada día, lo que es corroborado por profesores/as, quienes atestiguan este problema con similar magnitud en el colegio $(42,3 \%)$ y los salones de clase $(40,4 \%)$. En esto coinciden también los padres, que declaran haber sido testigos de la ocurrencia de insultos, garabatos, burlas y descalificaciones entre estudiantes muchas veces (35,4\%). Es interesante como esta tipología de conflicto escolar se encuentra extendida y reconocida por los actores, a la vez que evalúan la dimensión de Respeto como algo predominante en las relaciones. La violencia verbal, estrechamente vinculada con agresiones sociales como ley del hielo y psicológicas como la burla y el menoscabo (Postigo, González, Mateu, Ferrero y Martorell, 2008), lastra las relaciones interpersonales y dificulta que los mecanismos de resolución de conflicto se lleven a cabo mediante el diálogo, por lo cual el control de estas situaciones que significan malos tratos es eje fundamental para las estrategias de intervención escolar.

Bajo el supuesto que la percepción de organización, seguridad y respeto se configura como respuesta al nivel de injerencia y proximidad que tienen los miembros de la comunidad escolar, el análisis del OIC-Clima de convivencia escolar se desagregó con base al contexto o espacio de interacción, según refiera al salón de clases o al colegio, tal y como muestra el Tabla 4.

TABLA 4

Percepción de ambientes que componen clima de convivencia según nivel educativo y espacio

\begin{tabular}{|c|c|c|c|c|c|c|c|}
\hline & & \multicolumn{3}{|l|}{$8^{\circ}$ básico } & \multicolumn{3}{|l|}{$2^{\circ}$ Medio } \\
\hline & Ambiente & Estudiantes & Profesor & Apoderado & Estudiantes & Profesor & Apoderado \\
\hline \multirow[t]{2}{*}{ Áulico } & $\begin{array}{l}\text { - } \\
\text { Organizado } \\
\text { Respeto } \\
\text { - } \\
\text { Seguro }\end{array}$ & $\begin{array}{l}.669,678 \\
.548\end{array}$ & $\begin{array}{l}.678 \\
.777 \\
.622\end{array}$ & --- & $\begin{array}{l}.658,680 \\
.515\end{array}$ & $\begin{array}{l}.672 \\
, 776 \\
620\end{array}$ & --- \\
\hline & $\begin{array}{l}\text { - } \\
\text { Organizado } \\
\text { - } \\
\text { Respeto } \\
\text { - } \\
\text { Seguro }\end{array}$ & $\begin{array}{l}.723,738 \\
.808\end{array}$ & $\begin{array}{l}.672 \\
.816 \\
.811\end{array}$ & $\begin{array}{l}.657,840 \\
.748\end{array}$ & $\begin{array}{l}599,701 \\
.800\end{array}$ & $\begin{array}{l}.602 \\
.794 \\
.759\end{array}$ & $\begin{array}{l}.783,849 \\
.597\end{array}$ \\
\hline
\end{tabular}

Elaboración propia basada en datos aportados por Agencia Calidad de la Educación (2014).

Si bien varios reportes de investigaciones recientes acerca de violencia y conflicto escolar señalan que es el espacio extra aula donde se producen mayormente los actos de amenazas y/o golpes (Tijmes, 2012; Gázquez, Pérez y Carrión, 2011), en lo que a nuestros datos respecta, el salón de clases parece un sitio menos seguro que el colegio. El aula, unidad principal de convivencia y enseñanza-aprendizaje para los y las estudiantes, es un lugar donde se aprende a cómo reconocer y tratar al resto que no pertenece a la familia, donde estos actores se refugian y requieren con mayor acuciosidad sentirse parte y ser reconocidos/as, por ende, donde primeramente se ponen en marcha los mecanismos beligerantes de la socialización (Lomas, 2007).

Respecto de la unidad educativa, la Organización es para el estudiantado la dimensión peor evaluada (,599). En específico, son las acciones que desarrolla el profesorado, personal directivo o inspectores frente a las que se muestran más disconformes, y cómo ellos dan a conocer el reglamento de acciones para hacer frente al maltrato o intimidación (39,5\%). Este desconocimiento podría impedir que se emprendieran medidas contra la violencia observada o vivida; no saber dónde asistir, con quién hablar y la incertidumbre de quiénes se enterarán mitiga la posibilidad de informar problemas, especialmente de violencia, y más de reconocerse 
como víctima de bullying, pues la agresión no solo tiene una repercusión personal de angustia, sino también colectiva sobre cómo percibirá mi situación el resto.

En cuanto a tipologías de actor educativo, el profesorado muestra una mejor valoración del establecimiento que de las aulas ( $8^{\circ} \mathrm{b}=$ Aula, 692 vs Colegio ,766; $2 \mathrm{~m}=$ Aula ,689 vs Colegio ,718), excepto en lo que se refiere a Organización, donde manifiestan que las posibilidades de control son mejores. Con esto se esclarece la actitud antes hipotetizada de los profesores/as hacia el aula y alumnado como fuente de indisciplina, y que según los datos podemos aceptar parcialmente. Estar a cargo del salón no impide efectuar críticas al cumplimiento que tienen las normas dentro del espacio, sin embargo, éstas no podrían ser más agudas que las emitidas sobre el conjunto, lo que sucede fuera de mi control y corresponde a otros, es decir, puestos a evaluar los profesores/as aceptan que la Organización del aula está mal, pero no peor que la presente en el colegio. Percepción docente que ha sido documentada con anterioridad, que se tacha de autocomplaciente y explica debido al eventual cuestionamiento que las críticas hacia el curso producirían, tanto en su labor de educador como de regulador (Gázquez et ál. 2009; Castro et ál. 2012).

$\mathrm{Al}$ acotar los análisis al alumnado que más puede discernir respecto a fenómenos de convivencia (Tabla 5), sorprende que la violencia no sea la peor evaluada, puesto que la investigación sociológica en educación se ha encargado de dar cuenta de cómo el pensar, sentir y actuar vinculado a la clase social de pertenencia afecta las habilidades sociales y relacionales de los y las estudiantes (Bourdieu y Passeron, 1996; Cornejo y Redondo, 2007). En nuestro país la violencia se presenta con mayor frecuencia e intensidad en los establecimientos municipales, independiente del nivel y curso (Bellei, 2013; Canales y De los Ríos, 2009). En particular, son la exposición a las agresiones físicas y la deserción los problemas que mayormente deterioran estos climas, además de las diferencias en el capital cultural familiar que deben enfrentar los profesores/as a la hora de dictar los contenidos curriculares.

TABLA 5

Percepciones clima de convivencia y sus ambientes según variables de contexto en estudiantes de $2^{\circ}$ Medio

\begin{tabular}{|c|c|c|c|c|c|c|c|c|}
\hline & \multicolumn{4}{|c|}{ Estudiantes } & \multicolumn{4}{|c|}{ Profesorado } \\
\hline & $\mathrm{CCE}$ & $\mathrm{AR}$ & $\mathrm{AO}$ & AS & $\mathrm{CCE}$ & $\mathrm{AR}$ & $\mathrm{AO}$ & AS \\
\hline $\begin{array}{l}\text { Dep. Admin. } \\
\text { - Municipal } \\
\text { - Part. Subv. } \\
\text { - Part. } \\
\text { Pagado }\end{array}$ & $\begin{array}{l}.665 \\
.689 \\
.701\end{array}$ & $\begin{array}{l}.672 \\
.697 \\
.704\end{array}$ & $\begin{array}{l}.613 \\
.636 \\
.624\end{array}$ & $\begin{array}{l}.688 \\
.755 \\
.798\end{array}$ & $\begin{array}{l}.666 \\
.718 \\
.775\end{array}$ & $\begin{array}{l}.749 \\
.792 \\
.835\end{array}$ & $\begin{array}{l}.593 \\
.645 \\
.701\end{array}$ & $\begin{array}{l}.638 \\
.697 \\
.770\end{array}$ \\
\hline $\begin{array}{l}\text { Sexo - } \\
\text { Hombre - } \\
\text { Mujer }\end{array}$ & $\begin{array}{l}.681 \\
.686\end{array}$ & $\begin{array}{l}.689 \\
.693\end{array}$ & $\begin{array}{l}.619 \\
.638\end{array}$ & $\begin{array}{l}.668 \\
.650\end{array}$ & $\begin{array}{l}.709 \\
.711\end{array}$ & $\begin{array}{l}.780 \\
.790\end{array}$ & $\begin{array}{l}.636 \\
.637\end{array}$ & $\begin{array}{l}.691 \\
.688\end{array}$ \\
\hline $\begin{array}{l}\text { GSE - Bajo - } \\
\text { Alto }\end{array}$ & $\begin{array}{l}.672 \\
.702\end{array}$ & $\begin{array}{l}.681 \\
.705\end{array}$ & $\begin{array}{l}.629 \\
.624\end{array}$ & $\begin{array}{l}.649 \\
.701\end{array}$ & $\begin{array}{l}.644 \\
.776\end{array}$ & $\begin{array}{l}.729 \\
.836\end{array}$ & $\begin{array}{l}.570 \\
.703\end{array}$ & $\begin{array}{l}.612 \\
.771\end{array}$ \\
\hline
\end{tabular}

\footnotetext{
Elaboración propia basada en datos aportados por Agencia Calidad de la Educación (2014).

$\mathrm{Dep}=$ Dependencia; Admin = Administrativa; $\mathrm{CCE}=$ Clima de convivencia escolar; $\mathrm{AR}=$ ambiente de respeto; $\mathrm{AO}=$ ambiente organizado; $\mathrm{AS}=$ ambiente seguro; Part= particular; $\mathrm{Subv}=$ subvencionado; $\mathrm{GSE}=\mathrm{Grupo}$ Socioeconómico.
}

Al diferenciar por sexo, la evaluación del clima de convivencia entre estudiantes difiere, siendo las mujeres quienes reportan convivir en un mejor clima $\left[t_{\text {ind }}(178160)=9,273, \mathrm{p}<, 01\right]$; mientras que entre docentes la percepción es similar $[t$ IND $\quad(5921)=, 614, \mathrm{p}>, 05$; N.S ] La menor valoración que las estudiantes hacen del ambiente seguro puede estar asociado con que el mayor tipo de violencia reportada por este grupo de actores a nivel general es la de tipo verbal. No obstante la representación social del conflicto en la escuela posiciona al hombre como agresor y víctima, este es un fenómeno extensible al género femenino, en el cual se presenta de forma relacional-social, siendo sus principales acciones el rechazo y los insultos (Postigo, et ál. 2008). 
El profesorado muestra evaluaciones relativamente más altas según pertenezcan a establecimientos particulares o sean mujeres. Estas pequeñas diferencias, en razón que se circunscriben a percepción, pueden estar relacionadas con la mayor sensibilidad que estas tienen al esfuerzo y dedicación del estudiantado, más allá de la evaluación puntual de su comportamiento en determinada ocasión. Quizá en nuestro caso, tal y como dan cuenta Castro et ál. (2012) en un estudio sobre percepción de fracaso y logro escolar, los y las docentes tomen menos en cuenta los conflictos a la hora de evaluar el clima áulico, enfocándose más en el rendimiento académico o las relaciones que entablan con los y las estudiantes para definir cuál es el estado de la convivencia.

El asistir a un establecimiento de bajos recursos y que atiende a población vulnerable demuestra ser, en cuanto significación estadística, perjudicial para el Clima de Convivencia según estudiantes, especialmente si se los compara con aquellos donde pulula el capital en sus múltiples dimensiones $\left[t_{\text {ind }}(67051)=-31,727\right.$, $\mathrm{p}<, 01]$; situación más aguda en el caso de los profesores/as $\left[t_{\text {ind }}(2314)=-18,970, \mathrm{p}<, 01\right]$, quienes en su labor pedagógica no solo deben lidiar con el permanente déficit de recursos, sino que también con eventos de violencia, deserción y/o desinterés de los y las estudiantes que atiende. Handicaps de arrastre de una precaria socialización familiar y reducida educación en valores y respeto hacia los demás (Yuste y Pérez, 2008), cuya presencia es factor de riesgo para la buena convivencia escolar.

\section{Conclusiones}

Bajo una perspectiva que diversifica la comprensión y medición de la calidad en educación en Chile, los Otros Indicadores de Calidad buscan posicionar el desarrollo social y personal del alumnado en el epicentro de la preocupación por mejorar el sistema educativo. En su temprano desarrollo, el Clima de convivencia emerge como la matriz de interacciones que posibilita conocer, desde la perspectiva de los actores de la comunidad escolar, cuál es la situación en que cotidianamente aprenden, no tan solo en términos de contenidos, sino también respecto a sentimientos de seguridad, estado organizacional del espacio y respeto existente entre alumnos/as y docentes.

Según hemos discutido y similar a estudios anteriores en la temática, este artículo evidencia que el conflicto es una característica inmanente en diversos grados al clima de convivencia, y que dependiendo del tipo de actor sus manifestaciones se atenúan o cobran preocupación. Sin embargo, sostenemos que la resolución dialógica abre la oportunidad para fortalecer las relaciones interpersonales y desarrollar habilidades de comunicación y empatía, especialmente en la infancia. Como ideales pedagógicos de la escuela, el aprender a vivir juntos y aprender a ser se construye también desde el enfrentamiento y superación de este tipo de problemáticas, a lo cual se suma la relevancia de intervenir las dimensiones de seguridad, organización y respeto.

En cuanto a valoraciones del Clima de convivencia escolar, los y las estudiantes de secundaria son los actores más críticos, tanto de las interacciones que sostienen con sus compañeros como las desplegadas con profesores/as. El conflicto entre pares, principalmente de índole psicológica y por medios verbales, y la ausencia de reglas y normas conocidas, respetadas y aplicadas en el salón de clases, adquieren relativa habitualidad según la percepción estudiantil, todo lo cual deteriora el clima de convivencia, así como las condiciones en que se suscita el aprendizaje.

Si bien la relación profesor/a-alumno/a no está exenta de conflictos, estos están vinculados a la disciplina y el orden más que a la agresión, insultos o faltas de respeto. La jerarquía y rol que tiene instituido el personal docente lo previene, hasta cierta medida, de ser objeto de convivencia hostil. Los educadores son los que mejor evalúan el clima de convivencia, en especial cuando se refiere al aula. Al ser el salón de clase un espacio físico que define claramente su responsabilidad, la valoración que otorgan a los ambientes de Organización y Seguridad en él pueden ser producto de una actitud autocomplaciente con arreglo a expectativas más que a la realidad, o bien ser el reflejo de la efectiva posibilidad de actuar como ente regulador entre las interacciones 
que se atribuye. Al pronunciarse sobre el espacio extra-aula su evaluación positiva se mantiene, lo que puede explicarse por su diluida responsabilidad en este contexto, así como las restricciones y límites para observar lo que sucede fuera del salón de clases.

Los apoderados/as por su parte exhiben valoraciones significativamente mejores que el resto de actores. Su nivel de injerencia es escaso, así como la calidad de sus redes de información, generalmente esporádicas y sesgadas, cuya principal fuente son los relatos de sus propios hijos e hijas y las puntuales reuniones de padres y madres. A esta acotada participación, institucionalizada por la escuela, se suma la costumbre de delegar al profesorado la responsabilidad de cuidar y vigilar a los y las estudiantes.

En cuanto a los espacios, el aula parece ser un lugar significativamente menos seguro que el establecimiento independiente del tipo de actor y nivel educativo consultado. En términos comparativos el salón de clases establece una mayor proximidad física y cotidianidad relacional, cuya inmediatez y recurrencia condiciona la exposición a conflictos y episodios de agresión que pueden gatillar estas evaluaciones, las que se evidencian particularmente negativas en actores pertenecientes a establecimientos de conformación socioeconómica vulnerable.

Por tanto, la problematización que deriva de la atención al Clima de Convivencia, tanto por su nueva operacionalización como tipo de evaluación, da cuenta de la necesidad de fortalecer en la educación su función socializadora. Los aportes de este OIC a la profundidad del análisis, el espacio que abre a las particularidades de las unidades educativas y la diversificación que hace de los componentes del fenómeno, tributan directamente al cambio en la comprensión de la calidad y precisión de las políticas educativas. Así mismo, los resultados aquí expuestos a nivel de seguridad, organización y respeto percibidos por los actores educativos permiten precisar objetivos en intervención, planteando la discusión de cómo lograr efectos holísticos en su abordaje respetando la perspectiva compleja que permitió su operacionalización. En suma, el clima de convivencia es la matriz de relaciones entre agentes, y el resguardo de la seguridad no puede obviar la organización de los grupos ni el respeto entre personas; triada indispensable de tutelar si lo que se pretende es lograr cambios colectivos vinculados a la educación ciudadana e individuales como la autoestima y la motivación.

\section{REFERENCIAS}

Agencia Calidad de la Educación. (2014). Resultados SIMCE y Cuestionarios de contexto. Santiago de Chile: Ministerio de Educación. Recuperado de https://www.agenciaeducacion.cl/

Barca, A., Almeida, L., Porto, A., Peralbo, M. y Brenlla, J. (2012). Motivación escolar y rendimiento: impacto de metas académicas, de estrategias de aprendizaje y autoeficacia. Anales de psicología, 8(3), 848-859.

Bellei, C. (2013). El estudio de la segregación socioeconómica y académica de la educación chilena. Estudios Pedagógicos, 39(1), 325-345.

Bourdieu, P. y Passeron, J. C. (1996). La Reproducción: elementos para una teoría del sistema educativo. México: Fontamara.

Bourdieu, P. (2016). La distinción: criterio y bases sociales del gusto. Barcelona: Taurus.

Canales, A. y De los Ríos, D. (2009). Retención de estudiantes vulnerables en la educación universitaria. Revista Calidad en la educación, 50-83.

Carabaña, J. (2016). El informe Coleman cincuenta años después. Revista de la Asociación de Sociología de la Educación, 9(1), 1-16.

Carrasco, C. y Trianes, V. (2010). Clima social, prosocialidad y violencia como predictores de inadaptación escolar en primaria. European Journal of Education and Psychology, 3(2), 229-242.

Castro, P., General, F., Jofré, R., Saéz, N., Vega, Á. y Bortolluzi, M. (2012). Teorías subjetivas de profesores sobre la motivación y sus expectativas de éxito y fracaso escolar. Educar em revista, (46), 159-172. 
Cervini, R., Dari, N. y Quiroz, S. (2015). Género y rendimiento escolar en América Latina. Los datos del SERCE en Matemática y Lectura. Revista Iberoamericana de Educación, 68, 99-116.

Coleman, J. (1968). Equality of Educational Opportunity. Integrated Education, 6(5), 19-28.

Conejeros, M. L., Rojas, J. y Segure, T. (2010). Confianza: un valor necesario y ausente en la educación chilena. Perfiles educativos, 32(129), 30-46.

Cornejo, R. y Redondo, J. (2007). Variables y factores asociados al aprendizaje escolar. Una discusión desde la investigación actual. Estudios Pedagógicos, 33(2), 155-175.

Del Rey, R., Ortega, R. y Feria, I. (2009). Convivencia escolar: fortaleza de la comunidad educativa y protección ante la conflictividad escolar. Revista interuniversitaria de formación del profesorado, (66), 159-180.

Flórez, M. T. (2013). Análisis crítico de la validez del sistema de medición de la calidad de la educación (SIMCE). Reino Unido: Universidad de Oxford.

Fueyo, E., Martín, M. y Dapelo, B. (2010). Personalidad eficaz y rendimiento académico: una aproximación integrada. Revista de orientación educacional, 24(46), 57-70.

García, J. y Pérez, M. (2008). Espacio Europeo de Educación Superior, competencias profesionales y empleabilidad. Revista iberoamericana de educación, 46(9), 1-12.

Gázquez, J., Pérez, M. C., Acién, F. y Fernández, M. (2009). Análisis de la convivencia escolar por los docentes europeos. Aula abierta, 37(2), 11-18.

Gázquez, J., Pérez, M. C. y Carrión, J. (2011). Clima escolar y resolución de conflictos según el alumnado: un estudio europeo. Revista de Psicodidáctica, 16(1), 39-58.

Gilbert, J. (2012). Introducción a la sociología. Santiago de Chile: LOM.

Guerra, C., Álvarez, D., Dobarro, A., Nuñez, J., Castro, L. y Vargas, J. (2011). Violencia escolar en estudiantes de educación secundaria de Valparaíso (Chile): comparación con una muestra española. Revista Iberoamericana de Psicología y Salud, 2(1), 75-98.

Leyton, D., Vásquez, A. y Fuenzalida, V. (2012). La experiencia de estudiantes de contextos vulnerables en diferentes instituciones de educación superior universitaria (IESU): resultados de investigación. Calidad en la educación, (37), 61-97.

Lomas, C. (2007). ¿La escuela es un infierno? Violencia escolar y construcción cultural de la masculinidad. Revista de Educación, (342), 83-101.

López, V., Carrasco, C., Morales, M., Ayala, Á., López, J. y Karmy, M. (2011). ndividualizando la Violencia Escolar: Análisis de Prácticas Discursivas en una Escuela Municipal de la Región de Valparaíso. Psykhe, 20(2), 7-23.

Martinic, S. (2002). La reforma educativa en Chile. Umbral, (8), 1-12.

Ministerio de Educación de Chile. (2009). Objetivos fundamentales y contenidos minimos obligatorios de la educación básica y media. Santiago de Chile: Ministerio de Educación.

Ministerio de Educación de Chile. (2013). Fundamentos otros indicadores de calidad. Santiago de Chile: Unidad de Currículum y Evaluación.

Ministerio de Educación de Chile. (2014). Otros indicadores de calidad educativa. Santiago de Chile: Unidad de Currículum y Evaluación.

Ministerio del Interior. (2006). Principales resultados del estudio nacional de violencia en el ámbito escolar. Santiago de Chile: Universidad Alberto Hurtado y Gobierno de Chile.

ONU. (2010). Objetivos de desarrollo del milenio. Informe 2010. Nueva York: Naciones Unidas.

Pereira, J. M. (2010). Consideraciones básicas del pensamiento complejo de Edgar Morin en la educación. Revista Electrónic@Educare,14(1),67-75.

Postigo, S., González, R., Mateu, C., Ferrero, J. y Martorell, C. (2008). Diferencias conductuales según género en convivencia escolar. Psicothema, 21(3), 453-548.

Román, M. y Murillo, J. (2011). América Latina: violencia entre estudiantes y desempeño escolar. Revista CEPAL, (104), 37-54. 
Serramona, J. (2004). Participación de los padres y calidad de la educación. Estudios sobre Educación, (6), 27-38.

San Juan, C. y Vozmedia, L. (2008). Mediación de la inseguridad y análisis del miedo al delito con sistemas de información geográfica. En M. Campo, L. Castaño, J. García, A. Bueno y P. Gómez, Perspectivas y enfoques sobre seguridad ciudadana (pp. 27-42). Bogotá: Cámara de Comercio de Bogotá.

Tijmes, C. (2012). Violencia y clima escolar en establecimientos educacionales en contextos de alta vulnerabilidad social de Santiago de Chile. Psybke, 21(2), 105-117.

Trautmann, A. (2008). Maltrato entre pares o "bullying": Una visión actual. Revista chilena de pediatria, 79(1), 13-20.

Yuste, N. y Pérez, M. D. (2008). Las cuestiones familiares como causa de la violencia escolar según los padres. European Journal of Educación and Psychology, 1(2), 19-27.

\section{Notas}

[1] Valores representan las categorías de respuesta Muy en desacuerdo y Desacuerdo.

\section{BY-NC-ND}

